MEETING REPORT

\section{International Transdisciplinarity Conference 2021}

\section{Creating spaces and cultivating mindsets}

for learning and experimentation

\author{
Christina Benighaus, Institut für Technikfolgenabschätzung und \\ Systemanalyse (ITAS) \& KIT-Zentrum Mensch und Technik (MuT), \\ Karlsruher Institut für Technologie (KIT), Karlstr. 11, 76133 Karlsruhe, DE \\ (benighaus@kit.edu) @0000-0002-5537-5593 \\ Richard Beecroft, KIT-Zentrum Mensch und Technik (MUT), \\ Karlsruher Institut für Technologie (KIT), 76131 Karlsruhe, DE \\ (richard.beecroft@kit.edu) (10 0000-0002-3170-5201
}

Die International Transdisciplinarity Online-Conference 2021 (ITD 21), organisiert vom Network for Transdisciplinary Research und dem Transdisciplinarity Lab der ETH Zürich, kam mit über 500 Teilnehmenden wuchtig daher. Entsprechend hoch waren unsere Erwartungen an die mehr als 200 Real-time-Sessions und Posterbeiträge. Auch inhaltlich hatte sich die Tagung den hohen Anspruch gesetzt, eine Plattform zu bieten ,für Engagement und Diskussionen, die transdisziplinäre Forschung, (Un-)Lernen und Praxis miteinander verbindet".

Das Thema war herausfordernd für eine Tagung, die ausschließlich online stattfinden konnte: ,Creating spaces and cultivating mindsets for learning and experimentation" (Räume schaffen und Denkweisen für Lernen und Experimentieren kultivieren). Wie wird eine Online-Tagung dem gerecht? Mit knappen, vorbereitenden Inputs schöpfte die Konferenz die digitalen Möglichkeiten für Texte, Bilder, Filme oder Narrative voll aus. Ebenso lohnte sich der erste Tag zur Vernetzung der transdisziplinären Nachwuchsforscher:innen, für die mehrtägige internationale Reisen auch ohne Pandemie oft kaum organisierbar sind. Das richtige ,Tagungsgefühl ' wollte aber trotz aller inspirierender Elemente im Programm nicht so recht aufkommen. Vielleicht war das Angebot zu breit gestreut oder können Online-Veranstaltungen dieses Tagungsgefühl einfach nicht bieten?

This is an article distributed under the terms of the Creative Commons

Attribution License CCBY 4.0 (https://creativecommons.org/licenses/by/4.0/)

https://doi.org/10.14512/tatup.30.3.82

Received: Oct. 20, 2021; revised version accepted: Nov. 05, 2021;

published online: Dec. 20, 2021 (editorial peer review)
Was die Online-Tagung ohne Reisekosten wiederum ermöglichte: Die Community ist international geworden. Dominierten in den Anfangszeiten des Netzwerks noch der deutschsprachige und nordamerikanische Diskurs, sind inzwischen Anzahl und kulturelle Hintergründe der Teilnehmenden stark gewachsen. Auch die außergewöhnlich sozial gestaffelten Konferenzgebühren ermöglichten die Teilnahme für viele, die wegen zu hoher Kosten außen vor geblieben wären. Insbesondere für die Diskussion von Nachhaltigkeitsinitiativen lohnte sich dieser internationale und interkulturelle Blick. Die Themen der Tagung waren so vielfältig wie die internationale Community der transdisziplinären (TD) Forschung. In vielen Sessions wurde vor allem um eine allgemeingültige Definition der TD-Arbeit gerungen, die kontextuellen Unterschiede und Gemeinsamkeiten der inter- und transdisziplinären Forschung präsentiert. Es scheint weiterhin ein langer, herausfordernder Weg zu sein, das Fachwissen aus den verschiedensten Wissensgebieten durch TD-Konzepte zu integrieren und einen Beitrag zur Koproduktion von Wissen zu leisten.

Die Vielfalt der präsentierten Fallstudien und Beispiele sowie das Engagement der Community waren begeisternd und mitreißend, allerdings blieben viele Diskussionen sehr nah an der Praxis. Theoriearbeit, also was denn dieses am Lernen orientierte Selbstverständnis der transdisziplinären Community bedeutet, blieb eher im Hintergrund. Ebenso hätten Bezüge auf einschlägige Theoriegebäude, die sich mit Lernprozessen beschäftigen, wie zum Beispiel Psychologie, Bildungstheorie oder Innovationstheorien, stärker hervorgehoben werden können. Mit der Öffnung hin zu Bildungsthemen werden auch Anschlüsse an Diskurse zu inneren Dimensionen der Nachhaltigkeit - z. B. Werte- und Bewusstseinswandel - einfacher, die aus einem klassischen Wissenschaftsverständnis heraus schwer zugänglich

\section{Wie können wir Wissen als valide qualifizieren, das in trans- disziplinären Settings erzeugt wird?}

sind. Aber es ging auch um die wissenschaftliche Glaubwürdigkeit integrierten Wissens, das in einer solchen TD-Forschung produziert wird. Zentrale Fragen lauteten beispielsweise: „Wie stellen wir die wissenschaftliche Nachvollziehbarkeit von Methoden zur Wissensintegration und der Ergebnisse solcher Methoden sicher?“ Oder, ,Wie können wir Wissen als valide qualifizieren, das in transdisziplinären Settings erzeugt wird?

Und eine gute Nachricht: Bildung ist im Diskurs zur Transdisziplinarität angekommen! Dies nicht mehr nur in dem Sinne, dass Forschungsprozesse durch Lehrinitiativen flankiert werden, sondern dass die transdisziplinäre Forschung auch grundlegend als Lernprozess zwischen unterschiedlichen Akteur:innen, Perspektiven, Systemen oder Institutionen verstanden wird. 
Dabei betonte das Tagungsthema nicht, wie es sonst leicht passiert, die Lernerfolge bzw. deren Messung, sondern die genuin bildungswissenschaftlichen Fragestellungen: also welche Rahmen für Lernprozesse geschaffen werden sollten und wie sich die Geisteshaltung, das Mindset, der Beteiligten auf transdisziplinäre Prozesse einstimmen lässt.

Experimentieren ist zu einem systematischen Ankerpunkt in der transdisziplinären Methodologie geworden. Das ist keineswegs selbstverständlich für einen Diskurs, der in der Anfangszeit gerade durch das Beharren auf das systematische Erzeugen von Wissen erfolgreich wurde und vielfach auf der Ebene von Wissensintegration und Aushandlung, aber nicht primär auf der Ebene von Intervention und Experiment agierte. Mit den Bezügen auf die transformative Forschung und der Entstehung von längerfristigen Kooperationsstrukturen, die praktische Transformationen erleichtern (living labs, transformative innovation labs, Reallabore und verwandte Ansätze) wurden bei dieser Tagung wesentliche Ankerpunkte gelegt.

Neben diesen neuen Schwerpunkten blieben aber auch eine Reihe Themen sichtbar, von denen wir für die TD-Forschung hoffen können, dass sich in den nächsten Jahren Lösungen abzeichnen:

- Wie evaluiert man die Wirksamkeit und die wissenschaftliche Qualität transdisziplinärer Prozesse?

- Wie gelingt transdisziplinäre Koproduktion von Wissen statt eines immer noch währenden multidisziplinären Dialoges?

- Wie können digitale Methoden und Werkzeuge die TD-Arbeit sinnvoll unterstützen?

- Wie muss sich die Rolle der TD-Forschung um eine nachhaltige Entwicklung in der Gesellschaft in den nächsten Jahren verändern?

- Wie gelingt die Förderung wissenschaftlicher Karrieren in der transdisziplinären Forschung, solange Promotionsordnungen etc. disziplinär organisiert sind?

- Wie gelingt die Finanzierung von Forschungsprojekten zwischen den Domänen großer Forschungsförderer?

Gemeinsam mit der intensiven Diskussion von methodologischen Fragen, die diesem Diskurs eigen ist, entsteht Selbstreflexion. Resümee: Eine Tagung allein kann die TD-Landschaft nicht weiterentwickeln. In den nachfolgenden Konferenzen wäre eine stärkere Orientierung auf die Inhalte und Wirkungen transformativer Forschung gewinnbringend und es wird noch viele Diskurse dazu geben müssen. Was mit der ITD 21 auf jeden Fall gelungen ist, ist weltweit denen eine geistige Heimat anzubieten, die bislang eher als Einzelkämpfer:innen transdisziplinär unterwegs waren und sich jetzt in einer großen Gemeinschaft der transdisziplinären Arbeit wiederfinden.

\author{
LETTER \\ Gegendarstellung \\ Uwe Schimanks Fehldeutungen \\ der Echtzeitgesellschaft

\begin{abstract}
Johannes Weyer, TU Dortmund, Fakultät Sozialwissenschaften, (johannes.weyer@tu-dortmund.de) (10) 0000-0002-0181-8723
\end{abstract} \\ Fachgebiet Techniksoziologie, Otto-Hahn-Str. 4, 44227 Dortmund, DE
}

In Jg. 30, Heft 1 (2021) der Zeitschrift TATuP ist eine Rezension meines Buchs „Die Echtzeitgesellschaft“ erschienen, die voller Fehler und Falschbehauptungen ist. Uwe Schimank behandelt Themen, die in dem Buch nicht vorkommen, etwa „Ambivalenz“, „Transintentionalität“, „,Reflexionsentlastung“, ,großtechnische Systeme zweiter Ordnung " und vieles anderes mehr. Andere Themen deutet er in einer Weise um, die den in meinem Buch dargestellten Forschungsergebnissen diametral widersprechen. Zudem versäumt er es, die von mir entwickelten zentralen Gedanken und Argumente wie auch die Ergebnisse der von mir durchgeführten empirischen Studien zu erwähnen beziehungsweise kritisch zu würdigen.

\section{Unerwähntes, Gegenteiliges und Übergangenes}

Im Detail: Ausgiebig und an mehreren Stellen seiner Rezension breitet Schimank die Trias ,sachlich, sozial, zeitlich“ als analytischen Rahmen einer Befassung mit smarter Technik aus. In meiner „Echtzeitgesellschaft“ findet diese Trias nur ein einziges Mal und eher en passant Erwähnung (S. 17). Mein analytischer Rahmen ist die Unterscheidung der drei Ebenen Mikro, Meso und Makro wie auch das Zusammenspiel dieser drei Ebenen in Mikro-Meso-Makro-Modellen.

Jeweils ein Kapitel des Buchs befasst sich mit der MenschMaschine-Interaktion (Mikro), dem Risikomanagement in Organisationen (Meso) sowie der Steuerung komplexer Systeme (Makro). Davon erfährt die Leser:in in Schimanks Rezension nichts. Auch dass ich zu allen drei Themenbereichen Ergebnisse umfangreicher empirischer Studien vorgelegt habe, bleibt unerwähnt.

Ein zweites Argument, das Schimanks Rezension prägt, ist das des Kontrollverlusts als Folge des Umgangs mit smarter Technik. In der Tat spielt dieses Thema in meinem Buch eine wichtige Rolle. Allerdings zeigen die empirischen Studien z.B.

This is an article distributed under the terms of the Creative Commons

Attribution License CCBY 4.0 (https://creativecommons.org/licenses/by/4.0/) https://doi.org/10.14512/tatup.30.3.83

Received: Jun. 28, 2021; revised version accepted: Aug. 03, 2021

published online: Dec. 20, 2021 (editorial peer review) 